\title{
When phonological neighborhood density both facilitates and impedes: Age of acquisition and name agreement interact with phonological neighborhood during word production
}

\author{
Hossein Karimi ${ }^{1} \cdot$ Michele Diaz $^{1}$ \\ Published online: 5 May 2020 \\ (C) The Psychonomic Society, Inc. 2020
}

\begin{abstract}
The number of similar-sounding words that a target word has, or its Phonological Neighborhood Density (PND), has been shown to influence word production. However, reported results are sometimes inconsistent, with studies showing facilitation, inhibition, and null effects of phonological neighbors. These mixed results may be due to the influence of other factors beyond PND. We investigated the potential interactions between a broad measure of PND (bPND), and age of acquisition (AoA), frequency, and name agreement in order to see if the effect of bPND varies as a function of these three variables. We examined the effect of bPND on the latency of picture naming and observed significant interactions between bPND and AoA such that bPND facilitated lexical retrieval for words that were acquired early, but inhibited retrieval for words acquired later in life. We hypothesize that lexical retrieval difficulty ultimately depends on the activation level of the target word's phonological representations relative to the activation levels of its neighbors' phonological representations. When phonological features of the target word are weakly activated (i.e., late AoA), and bPND is high, the neighbors' activation may overshadow the target's, impeding target retrieval. However, when the target's phonological representation is strongly activated, the activation of the neighbors might not exceed that of the target, thereby supporting phonological retrieval. We also observed interactions between bPND and name agreement such that increasing bPND led to faster reaction times (RTs), particularly when name agreement was lower, suggesting that bPND may also facilitate word retrieval when lexical competition is high.
\end{abstract}

Keywords Phonological Neighborhood Density $\cdot$ Age of acquisition $\cdot$ Frequency $\cdot$ Name agreement $\cdot$ Language production

\section{Introduction}

Producing a word involves retrieving the semantic, lexical, and phonological features of that word from semantic memory. Because human language makes use of a limited number of sounds (phonemes and vowels) to create an unlimited number of words, multiple word forms often sound similar to one another. When words are so similar that they vary only by one sound, they constitute a word's phonological neighbors. For example, the word "cat" has many phonological neighbors, such as "mat," "bat," "rat," "cut," "cab," "cap,"

Electronic supplementary material The online version of this article (https://doi.org/10.3758/s13421-020-01042-4) contains supplementary material, which is available to authorized users.

Michele Diaz

mtd143@psu.edu

1 Department of Psychology, The Pennsylvania State University, 356 Moore Building, University Park, PA, USA "caught," etc., whereas the word "scissors" has few, if any, neighbors. Thus, words are said to vary in terms of the number of phonological neighbors they have, namely, their Phonological Neighborhood Density (or PND; Landauer \& Streeter, 1973). Interestingly, previous research has shown that producing a word involves activation of not only the phonological features of the target word but also the activation of its phonological neighbors. This is believed to occur as the target word activates its own phonological features, and this activation spreads to other words that share some of these features via feedback from phonological to lexical representations (e.g., Dell, 1986, 1988).

Interestingly, activation of the phonological features of neighboring words has been shown to have opposing effects across comprehension and production of the target word. Many studies have reported inhibitory effects of PND during language comprehension, with longer RTs for words from denser neighborhoods (e.g., Goldinger, Luce, \& Pisoni, 1989; Luce, Goldinger, Auer, \& Vitevitch, 2000; Luce \& Pisoni, 1998), and numerous studies have reported facilitatory 
effects of PND during language production, with faster RTs or fewer errors for words from denser neighborhoods (e.g., Baus, Costa, \& Carreiras, 2008; Gordon, 2002; Harley \& Brown, 1998; Middleton \& Schwartz, 2010; Pérez, 2007; Stemberger, 2004; Tsai, 2018; Vitevitch, 2002a; Vitevitch \& Sommers, 2003; see Vitevitch \& Luce, 2016, for a review). However, the reported results for the effect of PND are not always consistent even within language production. For example, some studies investigating the effect of PND on word production have reported inhibitory effects of PND (e.g., Frank, Tanenhaus, Aslin, \& Salverda, 2007; Newman \& German, 2005; Sadat, Martin, Costa, \& Alario, 2014; Vitevitch \& Stamer, 2006). Moreover, some studies have reported a null effect of PND on word production (e.g., Jescheniak \& Levelt, 1994; Tabak, Schreuder, \& Baayen, 2010). Thus, it is still an open question whether and how PND affects word retrieval during language production.

One issue that may have contributed to these discrepant findings is that early investigations into the effect of PND on word production did not control for the potential effects of other factors that are known to influence word production (such as Age of Acquisition (AoA), frequency, and name agreement, e.g., Best, 1995; Goldrick, Folk, \& Rapp, 2010; Vitevitch, 1997). While more recent studies controlled for such factors either experimentally or through statistical techniques (see Middleton \& Schwartz, 2010; Pérez, 2007; Sadat et al., 2014, for examples), for the most part, studies have focused on the main effect of PND while controlling for these other variables, but have not tested how PND might interact with them. For instance, although Sadat et al. (2014), controlled for a whole host of variables that could influence word production, they did not examine potential interactions of these factors with PND. Thus, it is unknown whether and how PND might interact with other important factors that are known to affect word production. Moreover, many studies have only examined a limited sampling of words (e.g., CVC (consonant-vowel-consonant), monosyllabic words). It is critical to examine the potential interactions between PND and factors such as AoA, frequency, and name agreement, which have been shown to have considerable effects on language production in general (e.g., Oldfield \& Wingfield, 1965; Paivio, Clark, Digdon, \& Bons, 1989). In fact, the inconsistency between the previous findings on the effect of PND on word production might have arisen from ignoring these potential interactions.

Understanding the factors that interact with PND would provide valuable insight into how phonology interacts with lexical processing to contribute to language production. For instance, based on interactive models of language production, the effect of PND on word production is explained by arguing that bilateral feedback between phonological and lexical representations leads to activation of phonological neighbors, reinforcing the activation of both the lexical and the phonological features of the target word, and ultimately resulting in easier production (Dell, 1986, 1988; Dell \& Gordon, 2003). However, it is not well understood, under these models, whether and how a word's phonological activation (presumably reflected in its frequency or AoA), might interact with that of its phonological neighbors during retrieval. One possibility is that word retrieval difficulty is a function of the relative activation difference between a target and its neighbors such that retrieval is easier when a target's ultimate activation level is higher than its phonological neighbors, but more difficult when a target's activation level is lower than its neighbors (see Chen \& Mirman, 2012; Luce \& Pisoni, 1998; Mirman, Kittredge, \& Dell, 2010; Mirman \& Magnuson, 2008). Such differences in the relative activation between a target and its neighbors might depend on the target's higher resting (baseline) activation (i.e., before feedback from the phonological to lexical nodes is initiated), or due to that target's greater power to suppress any inhibitors once phonological-to-lexical feedback has started.

In addition to PND, there are a number of factors that have been shown to influence naming difficulty, including (but not limited to) AoA, frequency, name agreement, visual complexity, number of syllables, phonotactic probability, etc. Among these factors, AoA, frequency, and name agreement have shown some of the largest effects on naming difficulty (for examples, see Alario, Ferrand, Laganaro, New, Frauenfelder, \& Segui, 2004; Britt, Ferrara, \& Mirman, 2016; Carroll \& White, 1973; Sadat et al., 2014). Numerous studies have shown that words acquired earlier in life are produced faster than words acquired later in life (e.g., Alario et al., 2004; Carroll \& White, 1973; Barry, Morrison, \& Ellis, 1997; Belke, Brysbaert, Meyer, \& Ghyselinck, 2005; Pérez, 2007). Although there are several explanations for AoA effects, many of these accounts propose that AoA affects the retrieval of the phonological form of target words (e.g., Brown \& Watson, 1987; Navarrete, Pastore, Valentini, \& Peressotti, 2015; Perret, Bonin, \& Laganaro, 2014), and also that the phonological representations of words that are acquired earlier are more robust, and have lower activation thresholds (e.g., Gilhooly \& Gilhooly, 1979; Gilhooly \& Watson, 1981; also see Morton, 1969). AoA may also affect the strength of connections between semantic and phonological representations, with early-acquired words having stronger connections than late-acquired words (e.g., Ellis \& Lambon Ralph, 2000; Lambon Ralph \& Ehsan, 2006). Finally, early-acquired words might have stronger lateral inhibition, which could function to reduce activation of competitors, compared to late-acquired words (Belke et al., 2005; also see McClelland \& Rumelhart, 1981).

Similar to AoA, lexical frequency has also been shown to affect word production. Lexical frequency refers to how often a word is used (normally based on some corpus count). Critically, the frequency of a word has also been shown to 
strongly affect naming latencies of pictures of that word, with faster RTs for more frequent words (e.g., Alario et al., 2004; Gordon \& Kurczek, 2014; Oldfield \& Wingfield, 1965; Sadat et al., 2014). Frequency effects, similar to AoA effects, are often explained through activation and/or inhibition accounts. More frequent words have higher baseline activation in memory relative to less frequent words, and may inhibit competitors more strongly, and, as a result, may require less cognitive effort to be retrieved (e.g., Belke et al., 2005; Borowsky \& Masson, 1999; Forster, 1976; McClelland \& Rumelhart, 1981; Morton, 1969; Paap, McDonald, Schvaneveldt, \& Noel, 1987; Rubenstein, Garfield, \& Millikan, 1970).

In addition to name agreement and frequency, another important factor that has been shown to have a strong influence on picture naming is name agreement. Name agreement refers to the total number of names that could be used to name a picture. Pictures that elicit many different names are said to have low name agreement compared to those that elicit fewer names. Importantly, past research has shown that name agreement affects naming difficulty such that pictures that have low name agreement cause greater picture-naming difficulty compared to pictures that have high name agreement (e.g., Barry et al., 1997; Lachman, Shaffer, \& Hennrikus, 1974; Paivio et al., 1989; Snodgrass \& Yuditsky, 1996; Vitkovitch \& Tyrrell, 1995). This is argued to be due to lexical competition: When name agreement is low, there are multiple names for a certain concept, increasing lexical competition and selection demands, and making word retrieval more difficult. Conversely, when name agreement is high, fewer names, or even only one name, fits a given concept, lowering competition and selection demands among different possible labels and facilitating word retrieval. ${ }^{1}$

As mentioned above, there are empirical and theoretical motivations to investigate how PND might interact with AoA, frequency, and name agreement. With regard to AoA, previous research has shown that words acquired earlier are produced and recognized faster, suggesting that they may have higher baseline activations, stronger inhibitory weights for suppressing competitors, stronger phonological representations, and stronger semantic and phonological links. As such, one might expect an interaction between AoA and PND such that early-acquired, but not late-acquired, words benefit from dense phonological neighborhoods. This is because if the phonological features of early-acquired words are robust, they are less likely to be hindered by the activation of the phonological neighborhood, and the activation of relevant phonological features may play a supportive role. Conversely, if the phonological features of late-acquired words are only

\footnotetext{
${ }^{1}$ Note that in picture naming, variations in name agreement could arise from image properties, such as when a picture is not a good representation of the associated word (e.g., a bad picture of, say, a tiger), or it could stem from lexical properties such as when a picture has multiple acceptable names (e.g., couch and sofa). In the present experiment, we focus on the latter.
}

weakly activated, the activation of phonological neighbors may cause interference because the neighborhood's activation might exceed that of the target. Note that similar arguments also motivate investigating the potential interaction between frequency and PND.

There is also strong reason to expect an interaction between PND and name agreement. Specifically, past research has shown that phonological speech errors (e.g., sciences when sinuses was intended), phoneme substitutions (beach for peach), and tip-of-the-tongue states are more frequent for words with sparser neighborhoods (e.g., Harley \& Bown 1998; James \& Burke, 2000; Vitevitch, 1997; Vitevitch, 2002b; Vitevitch \& Sommers 2003). Moreover, there is evidence showing that aphasic speakers produce fewer semantic errors for words with denser phonological neighborhoods (Middleton \& Schwartz, 2010). These results provide support for the idea that PND might privilege the retrieval of a target word over competing words. Based on these results, one might expect PND to facilitate picture naming more when lexical competition is higher (i.e., low name agreement). This is because lexical retrieval should be more difficult when lexical competition is high, and if PND boosts the activation of the target, it could exert a greater influence when lexical competition is more intense.

In this study, we investigated the potential effect of PND on picture-naming accuracy and RTs. By examining interactions between PND, AoA, frequency, and name agreement, we also tested whether the potential effect of PND on word production depends on any of these three variables. Also, it is important to note that picture naming is a more semantically-driven task than reading aloud. Since many previous studies have employed the word reading paradigm (e.g., Munson, Swenson, \& Manthei, 2005; Taler, Aaron, Steinmetz, \& Pisoni, 2010; Vitevitch, 2002a; Vitevitch \& Luce, 1998), our results would also shed light on how PND might interact with other important determiners of lexical access when phonological retrieval is not aided by orthography. Finally, investigating interactions between PND, AoA, frequency, and name agreement would provide further insight into how lexical and phonological units interact during word production.

\section{Method}

This experiment was a combination of multiple picture norming experiments conducted at Pennsylvania State University between 2016 and 2018. The purpose of each experiment was to select suitable pictures for a particular experiment from among a larger set of pictures. Importantly, the instructions and the procedure for each norming experiment was identical (see below), which allowed us to pool the data into a combined data set and perform a meta-analysis. We extracted the following three indices of PND from the 
English Lexicon Project website (visit http://elexicon.wustl. edu/ for more information): The number of Phonological Neighbors with Homophones ("Phono_N_H"), the number of Phonological Neighbors without Homophones ("Phono $\mathrm{N}$ "), and the mean of the Levenshtein distances for the 20 closest phonological neighbors ("PLD"). Note that both Phono N and Phono N H reflect the number of words that differ from a target word by addition, deletion, or replacement of a single phoneme, and both measures reflect neighborhood size, whereas PLD reflects the phonological distance between a target and its closest 20 neighbors. Thus, Phono_N and Phono_N_H represent the neighborhood size for a target word, and PLD represents close neighbors' similarity to a target.

Because these measures are highly correlated, we made a composite measure of PND. Including PLD in the composite measure also allowed us to include a more sensitive measure of words that had no phonological neighbors $(n=372 ; 18,157$ data points). Although a target word may have no phonological neighbors (that, by definition, vary by one phoneme from the target), it may still vary in how many similar-sounding words exist in relation to it. Conversely, target words with the same or similar PLD values may vary in terms of the number of phonological neighbors that they have. Thus, existing measures of PND might not capture all the variation in the phonological space, particularly when assessing a broad, more naturalistic array of items as we did in the present study.

The composite PND measure was created by running a Principal Component Analysis to determine the number of underlying components for Phono_N, Phono_N_H, and PLD. The results of this analysis revealed that one single component accounted for more than $99 \%$ of the variance among these three variables. We then ran a Factor Analysis to extract the loadings for that factor as the composite PND measure. Since this composite measure goes beyond the traditional PND measure (i.e., words that differ from a target by one Levenshtein distance), and captures a broader space in the phonological network, we refer to it as bPND, standing for broad phonological neighborhood density. It is important to note that bPND's correlation values with Phono_N, Phono_N_H, and PLD were 99\%,99\%, and -67\%, respectively. Given that bPND is more strongly correlated with neighborhood size measures (Phono_N and Phono_N_H) than with the phonological similarity measure (PLD), this measure is naturally more strongly associated with neighborhood density than with overall phonological similarity. We discuss the potential implications of these correlations in the General discussion. The $\mathrm{R}$ code for calculating bPND is provided at the Open Science Framework website (https://osf.io/pdgs4/files/).

\section{Participants}

Two hundred and twelve (147 female and 65 male) participants from the participant pool of the Psychology Department at Pennsylvania State University took part in the experiment. They were $18-23$ years of age (mean age $=19.02$ years, $S D=$ 1.73), all right-handed, and native speakers of American English, reporting no language-related disorders.

\section{Materials}

The experimental materials for the analysis consisted of 2,048 photographs of everyday objects corresponding to 1,137 unique words. The discrepancy between the number of pictures and the corresponding words was due to the fact that pictures for a target word varied across different experiments. All of the pictures were obtained from established databases (Brodeur, Dionne-Dostie, Montreuil, \& Lepage, 2010; Brodeur, Guerard, \& Bouras, 2014; Moreno-Martinez \& Montoro, 2012) or found on the internet. The responses for 123 pictures, corresponding to 86 words, always constituted either acceptable alternatives (but never the intended name), or incorrect responses, and were excluded from the analyses. Furthermore, 24 words did not have entries in the English Lexicon Project database and were also excluded. Thus, the final analysis included data from 1,901 images corresponding to 1,027 words.

The experiments were programmed in E-Prime 2.0 software (Psychology Software Tools, Pittsburgh, PA, USA). Two independent microphones recorded the experimental sessions. One was connected to E-Prime and recorded the RT of the response to the picture. The second microphone recorded the entire experimental session and was later used to code accuracy and calculate name agreement.

\section{Procedure}

Each picture was preceded by a fixation cross that lasted 500 ms. Immediately after the cross disappeared, the picture was presented and the participant could speak their response. Reaction times were calculated as the time from the onset of the picture until a response was made. Pictures disappeared as soon as a response was spoken, or after 3,000 ms. Participants were asked to name them as quickly as possible while still responding accurately. They were also instructed to use the specific name whenever possible. For example, they were told that upon seeing a "robin," they should say "robin" rather than "bird." However, they were also reminded that if they did not know the specific name for a picture, using a generic name ("bird") would be acceptable. We did this to minimize the use of superordinate category names (e.g., "animal," "food"), and therefore to maximize the variety of responses across pictures. Participants were also encouraged to limit their responses to only one word (e.g., "car" but not "red car"). 


\section{Coding}

Since we were interested in the effect of phonological neighborhood density, it was critical that the phonological form of the response was identical to that of the intended name. Thus, for any given picture (e.g., plane), we coded only identical responses (plane) and plural responses (planes) as "accurate". ${ }^{2}$ Responses that were accurate given the picture but did not have the same phonological code as the intended response (e.g., airplane, jumbo jet, big plane) were coded as "acceptable alternative." Responses that were incorrect descriptions of the picture were coded as "inaccurate" (e.g., helicopter for plane; beet for carrot; screwdriver for wrench). We removed any responses that were produced unreasonably quickly (i.e., faster than $200 \mathrm{~ms}$ ), or were slower than 2.5 standard deviations plus the mean for each subject, which amounted to 3,196 data points (5\%) of the data. Thus, eventually, we had three types of analyzable responses for each picture: Accurate, Acceptable Alternative, and Inaccurate.

\section{Data analysis}

Although we only included responses that matched the intended name in the main analysis, in order to capture the potential effect of lexical competition during naming (e.g., Barry et al., 1997; Lachman et al., 1974; Paivio et al., 1989; Snodgrass \& Yuditsky, 1996; Vitkovitch \& Tyrrell, 1995), we computed $\mathrm{H}$-indices on the correct and acceptable alternative responses to determine name agreement for each picture (Snodgrass \& Vanderwart, 1980). The advantage of using Hindex rather than mean accuracy as a measure of name agreement is that H-index takes into account not only the correct items, but also considers both the number of acceptable alternatives for a given item and the distribution of alternative responses, which produces a more complete indicator of naming demands. It is important to note that unlike mean accuracy, lower values of $\mathrm{H}$-index indicate higher name agreement and higher $\mathrm{H}$-index values denote lower name agreement.

We performed two main sets of analyses with two separate dependent variables: Accuracy and RT. However, because the predictors of interest did not have any significant effects on accuracy, we only report the RT analyses in the main text. The full results of the accuracy analysis are reported in the Online Supplementary Material. For the RT analysis, we included only the RTs associated with accurate responses, trimmed as described above. Also, in order to approach normality, we applied a $\log$ transformation on raw RTs when running the statistical models. Importantly, we controlled for the potential effects of number of syllables, phonotactic probability, ${ }^{3}$ and visual complexity of the pictures in the RT analysis. Visual

\footnotetext{
${ }^{2}$ Note that the reverse was also true: When the intended word was plural (apples), the response was coded as accurate if it was in singular form (apple).
}

complexity for each image was obtained by computing each image's entropy, and using it as a proxy for complexity. The entropy of an image corresponds to its (average) "information" content (vs. redundancy), and has been shown to affect behavior (e.g., Berman et al., 2014; Kardan et al., 2015; Rosenholtz, Li, \& Nakano, 2007). Moreover, previous research has shown that subjective and computational measures of visual complexity (including entropy) are highly correlated (e.g., Madan, Bayer, Gamer, Lonsdorf, \& Sommer, 2018).

We used linear mixed-effects (lmer) models from the lme4 package (Bates, Maechler, \& Bolker, 2011) in R (version 3.4.3, R Development Core Team, 2018) to analyze the RT data. Linear mixed-effects models can account for the variability of the effects within random effects (e.g., subjects, pictures), and are therefore less susceptible to spurious results and provide more generalizable outcomes. Following Barr, Levy, Scheepers, and Tily (2013), we always attempted to keep the random effects maximal. Thus, we started with a model that included random intercepts for both subjects and pictures, as well as by-subjects random slopes for all the predictors and all the interactions (i.e., the "full" model). If this model failed to converge, we systematically simplified the random-effect structure by first removing the correlation between random intercepts and random slopes (i.e., the "no-correlation" model) and then removing the random intercepts but keeping the random slopes (i.e., the "slopes-only" model; see Barr et al., 2013). However, since none of these models converged, we had to simplify the random-effects structures further. Specifically, because we were interested in whether and how bPND might interact with AoA, frequency and name agreement, we retained the random slopes for all the 2-way and 3-way interactions between bPND and these three variables, and removed random slopes for all other predictors. This is the suggested course of action by Barr et al. (2013) in case of non-converging models, and ensures that the effects of interest (in our case, bPND and its interaction with AoA, frequency and name agreement) are most generalizable. We also retained the random intercepts for both subjects and pictures. ${ }^{4}$ The R syntax for the main statistical models for accuracy and RT analyses are shown in (1).

1) $\operatorname{lmer}(\log (\mathrm{RT}) \sim$ Name Agreement + AoA + Frequency + bPND:Name Agreement + bPND:AoA + bPND:Frequency + AoA:Frequency:bPND + AoA:Name Agreement:bPND + Frequnecy:Name Agreement:bPND + Number of Syllables + Phonotactic Probability + Visual Complexity+ (1+bPND:Name

\footnotetext{
${ }_{3}^{3}$ Phonotactic probability was computed using the web-based interface provided by the University of Kansas (https://calculator.ku.edu/phonotactic/English/ words), and by summing the position-specific biphone probabilities for each of the biphones in the target word.

${ }^{4}$ Note that we used random slopes for each picture $(n=1,901)$, not for each word $(n=1,027)$.
} 
Agreement + bPND:AoA + bPND:Frequency + AoA:Frequency:bPND + AoA:Name Agreement:bPND + Frequnecy:Name Agreement:bPND |Participant)+ (1|Picture), data=data)

Note that by-picture random slopes do not apply to our design because each picture necessarily has only one value for AoA, Frequency, Name Agreement, and bPND. In all analyses, all the predictors were standardized to bring them to the same scale, and the $p$ values were calculated using normal approximation (Barr et al., 2013). Also, whenever we found a reliable interaction between predictors, we conducted follow-up analyses by factorizing AoA, Frequency, or Name Agreement to better understand the nature of their interaction with bPND. We always split AoA, Frequency and Name Agreement into "Low"/“"Early" and "High"/“Late" levels with .5 as the cut-off point for AoA and Frequency, and -.5 for Name Agreement. For all the simple effect analyses, either the "full" or the "slopes-only" model always converged and we did not have to simplify the random effects structure any further (Barr et al., 2013). The full data along with the analysis script can be accessed at the Open Science Framework website via the following link: https://osf.io/pdgs4/files/.

\section{Results}

The results of the RT analysis are reported in Table 1 below. Consistent with previous findings, we observed significant main effects of AoA, Frequency, and Name Agreement, with

Table 1 Results of the reaction time analysis

\begin{tabular}{lllll}
\hline Predictor & $\beta$ & $S E$ & $t$ & $p$ \\
\hline Name agreement & .044 & .004 & 11.09 & $<.001$ \\
AoA & .072 & .004 & 18.00 & $<.001$ \\
Frequency & -.024 & .004 & -5.92 & $<.001$ \\
Number of syllables & -.010 & .004 & -2.30 & .02 \\
Visual complexity & .008 & .003 & 2.32 & .02 \\
Phonotactic probability & -.000 & .003 & -.22 & .82 \\
bPND & .001 & .005 & .20 & .84 \\
bPND:Frequency & .004 & .004 & .91 & .36 \\
bPND:AoA & .008 & .004 & 2.01 & .04 \\
$\quad$ bPND within Early AOA & -.015 & .004 & -3.94 & $<.001$ \\
$\quad$ bPND within Late AoA & .036 & .013 & 2.84 & .004 \\
bPND: Name agreement & -.008 & .003 & -2.14 & .03 \\
$\quad$ bPND within Low Name Agreement & -.028 & .005 & -5.56 & $<.001$ \\
$\quad$ bPND within High Name Agreement & -.018 & .005 & -3.35 & $<.001$ \\
bPND:Frequency:Name Agreement & .007 & .004 & 1.63 & .10 \\
bPND:AoA:Name Agreement & .000 & .004 & .20 & .84 \\
bPND:AoA:Frequency & -.002 & .004 & -.57 & .57 \\
\hline
\end{tabular}

reliably faster RTs for words acquired earlier in life, for more frequent words, and for pictures with higher name agreement (e.g., Barry et al., 1997; Lachman et al., 1974; Oldfield, \& Wingfield, 1965; Paivio et al., 1989; Snodgrass \& Yuditsky, 1996; Vitkovitch \& Tyrrell, 1995). The effect of Visual Complexity was also significant, with faster RTs for less complex pictures (e.g., Attneave, 1957; Humphreys, Riddoch, \& Quinlan, 1988). However, the effect of Phonotactic Probability was not statistically significant. Interestingly, the effect of Number of Syllables was significant but in the unexpected direction: Faster RTs for words with more syllables. ${ }^{5}$ Finally, neither the two-way interaction between Frequency and bPND nor any of the three-way interactions were statistically reliable. Importantly, however, although the main effect of bPND was not significant, this predictor reliably interacted with AoA and name agreement.

Follow-up simple-effect analyses investigated the effect of bPND for early-, and late-acquired words. Figure 1 illustrates this interaction. As can be seen in this figure and in the results table, the effect of bPND on RTs reliably varied based on the AoA of the target word. Specifically, for early-acquired target words, bPND decreased RTs (i.e., facilitated naming). However, for late-acquired words, bPND increased RTs (i.e., impeded naming).

We also followed up the two-way interaction between bPND and name agreement, by examining the effect of bPND within low-, and high-name agreement pictures. The results are illustrated in Fig. 2. As can be seen in this figure and the results table, the facilitatory effect of bPND was greater for low- than for high-name agreement pictures, although the direction of the effect was the same across name agreement. $^{6}$

\section{General discussion}

In a large-scale experiment, we analyzed data from 212 participants and 1,901 pictures corresponding to 1,027 words, varying in terms of their phonological neighborhood densities,

\footnotetext{
${ }^{5}$ It is important to mention that although our overall analyses revealed faster naming times for words consisting of more syllables, simple effects of length showed the opposite pattern: Faster RTs for shorter words. Additional analyses (see the Additional Analyses in the Online Supplementary Material) revealed that with the addition of other factors (e.g., Frequency and AoA) the effect of length reverses. This suggests that length may interact with one or more of our other factors, and these complex inter-relationships may be driving the reverse length effect seen in the overall model.

${ }^{6}$ Based on a suggestion by an anonymous reviewer, we also examined the two-way interaction between AoA and frequency, which turned out to be statistically significant, with the facilitatory effect of frequency being greater for early- than for late-acquired words. This analysis is reported in an R file named "Additional Analyses" provided in the Online Supplementary Material. We think this interaction is interesting and seems to suggest additive facilitatory effects of frequency and AoA on word retrieval. However, because interactions between AoA and frequency did not interact with PND, the focus of the current paper, we will not discuss this effect any further.
} 


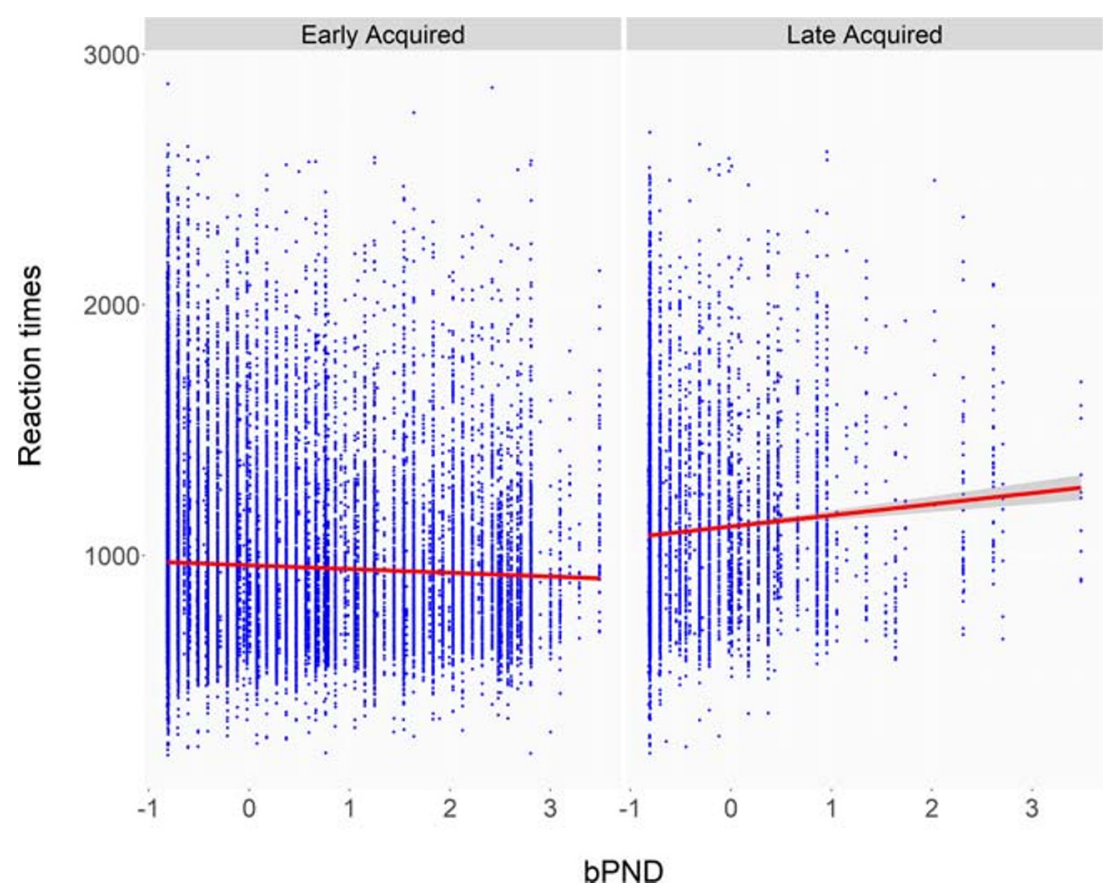

Fig. 1 bPND $\times$ AoA interaction. bPND decreased reaction times for early-acquired words, but increased reaction times for late-acquired words

AoA, frequencies, and name agreements. Although previous studies have looked at the main effect of PND controlling for other variables that have been shown to affect word retrieval, few studies have investigated the potential interaction of PND with these variables. Thus, in our study, while controlling for the main effects of AoA, frequency, name agreement, number of syllables, phonotactic probability, and visual complexity, we investigated how three important factors influencing word retrieval, namely, AoA, frequency, and name agreement, might interact with phonological neighborhood density. We incorporated a broad composite index of Phonological Neighborhood Density (bPND) that reflected both the number of phonological neighbors with and without homophones and the average Levenshtein distance of the 20 closest neighbors of a target word. We observed reliable interactions between bPND and AoA on RTs. Follow-up simple-effect analyses

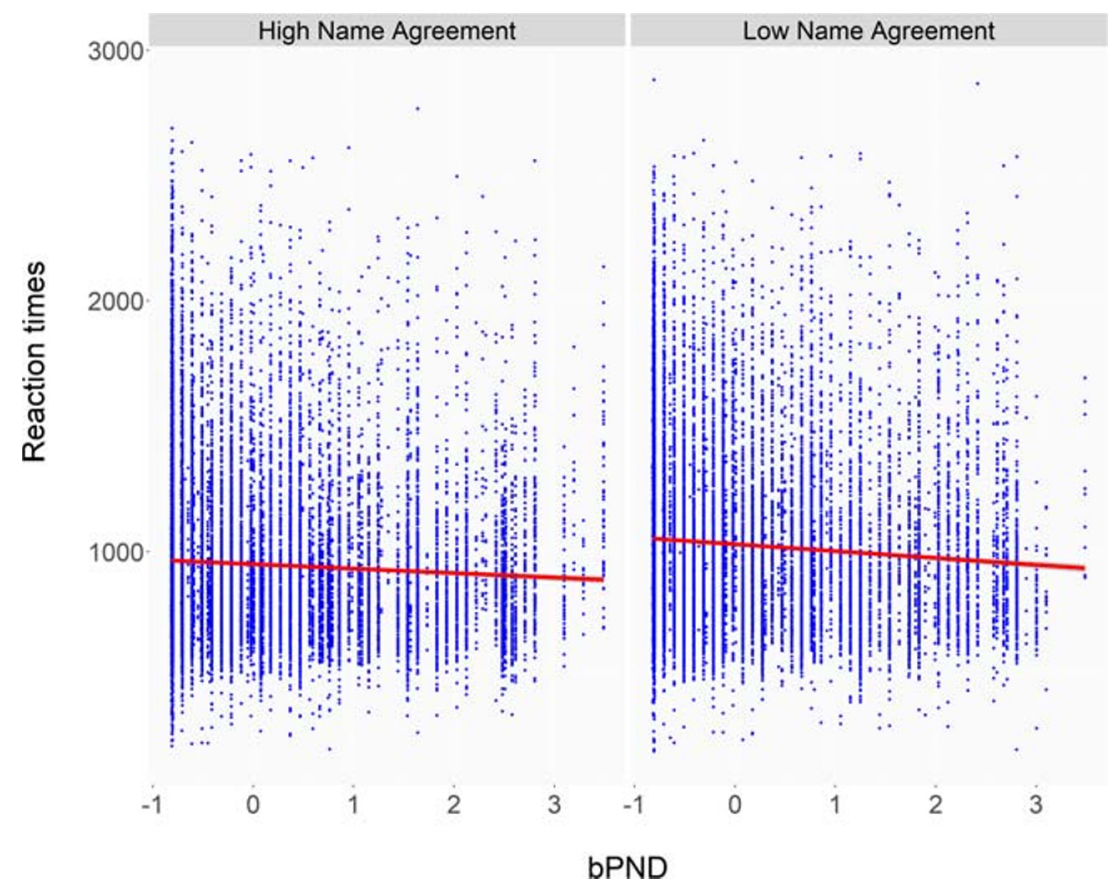

Fig. 2 bPND $\times$ Name Agreement Interactions for the reaction time analysis. The facilitatory effect of bPND is greater for low- relative to high-name agreement pictures 
revealed that bPND facilitated word retrieval for earlyacquired target words, but impeded word retrieval for lateacquired targets. bPND also reliably interacted with name agreement, such that increasing bPND speeded naming to a greater degree for pictures with lower name agreement. In what follows, we discuss the pattern of interactions between bPND and AoA, as well as bPND and name agreement.

\section{bPND $\times$ AoA interaction}

The bPND $\times$ AoA interaction in the RT analyses clearly shows that the effect of phonological neighborhood cannot be fully understood in isolation. More specifically, our results demonstrate that bPND has opposing effects depending on the AoA of the target word, facilitating word retrieval for earlyacquired words, but inhibiting word retrieval for lateacquired words. This interaction might explain the opposing results reported in the extant word production literature. As mentioned in the Introduction, previous studies have reported facilitatory, inhibitory, and null effects on word production (facilitatory: e.g., Baus et al., 2008; Gordon, 2002; Harley \& Brown, 1998; Middleton \& Schwartz, 2010; Perez 2007; Tsai, 2018; Vitevitch, 2002a; Vitevitch \& Sommers, 2003, inhibitory: Frank et al., 2007; Newman \& German, 2005; Sadat et al., 2014; Vitevitch \& Stamer, 2006, null: Jescheniak \& Levelt, 1994; Tabak et al., 2010). Our results cast new light on this issue by providing solid evidence for a conditional effect of phonological neighborhood. Assuming that AoA affects the strength of phonological representations in memory (e.g., Brown \& Watson, 1987; Navarrete et al., 2015; Perret et al., 2014), our results show that when the target's phonological representation is weaker (by virtue of being acquired later in life), the activation of the phonological neighbors may exceed that of the target, impeding the retrieval of the target. However, when a target's phonological activation is strong (by virtue of being acquired earlier in life), the relative activation of the neighborhood remains lower than the target, and consequently, the retrieval of the target can benefit from activation of items with similar phonological features, facilitating the target's retrieval. Such differences in relative strength of activation might arise from higher baseline activation levels for early- versus late-acquired words, or from stronger inhibitory connections to phonological competitors for early- than late-acquired words. In either case, our results clearly show that bPND can inhibit or facilitate word retrieval, depending on the strength of the phonological representation of the target relative to the collective activation of the phonological features of its neighbors.

Our results are consistent with previous research showing that AoA affects the strength of phonological representations (e.g., Brown \& Watson, 1987; Navarrete et al., 2015; Perret et al., 2014), and with the "attractor dynamics" model of lexical processing (e.g., Chen \& Mirman, 2012; Mirman,
Kittredge \& Dell, 2010; Mirman \& Magnuson, 2008; Spivey, 2007). Based on this account, each concept in semantic memory is viewed as a stable mental state, or an "attractor basin". Critically, semantic and phonological neighbors of a target word constitute other attractors that can influence word processing. Relevant to the current results, distant neighbors are argued to facilitate settling on the target basin (i.e., word retrieval) by causing the target basin to become broader, whereas near neighbors do not enlarge the size of the target basin and actually might function as competing basins, impeding the word retrieval process (see Mirman et al., 2010). Similarly, we can argue that when the relative activation of the target's phonological features is greater than the activation of its neighbors' phonological features, the neighborhood activation functions just like far neighbors, broadening the target basin and facilitating word retrieval. However, when the activation of the neighbors exceeds (or approaches) that of the target, competing basins are created, resulting in longer reactions times.

Our results also provide support for interactive models of language production by showing that feedback from phonological to lexical nodes affects word retrieval difficulty (e.g., Dell, 1986, 1988); if no activation was sent from phonological to lexical nodes, bPND should have had no effect on retrieval difficulty of targets words. However, we did observe a bPND effect, lending support to the existence of feedback connections from phonological to lexical nodes. Nonetheless, our results are agnostic to the possibility that such connections are mainly bottom-up in nature, or, alternatively, whether they are fully bilateral. Truly bilateral connections imply additional influence from lexical to phonological nodes after lexical representations have received activation from the phonological representations. One way to test such connections would be to include depth of semantic knowledge or vocabulary size as predictors. Although we did not collect such information from our participants in this study, this is an important question for future investigations. ${ }^{7}$

Despite this limitation, our results make important contributions to interactive models of language production (e.g., Dell, 1986, 1988) by suggesting that activation from phonological to lexical units may have opposing effects depending on the strength of a target's phonological representation (as reflected by its AoA) relative to that of its phonological neighbors. When the target's phonological activation is weaker than that of its neighbors, feedback from phonological nodes might overshadow the activation of the target, leading to inhibition. However, when the target's activation exceeds that of its phonological neighbors, activation from phonological representations may play a supportive role during word production.

\footnotetext{
${ }^{7}$ We thank an anonymous reviewer for bringing up the distinction between bottom-up versus bilateral connections between the phonological and lexical representations.
} 
Although effects may be due to differences in baseline, inhibitory, or lexical-semantic effects, one important implication of our results for interactive models is that feedback connections from phonological to lexical nodes do not always lead to the same result. Rather, such connections might be beneficial or detrimental to the retrieval of a target word depending on the ultimate relative activation between the target and its phonological neighborhood.

Given the high amount of overlap between AoA and frequency (i.e., early-acquired words tend to be more frequent), one question that might arise is why bPND interacted with AoA but not with frequency. One reason for this pattern of results could be that although AoA and frequency are fairly well correlated (-38\% in our dataset), they do not necessarily represent the same underlying construct. AoA has been shown to exert an independent influence on word retrieval (e.g., Izura et al., 2011), and others have shown that the effect of frequency disappears when AoA is taken into account (Morrison, Ellis, \& Quinlan, 1992). Moreover, past research has shown that the AoA effect is independent of both cumulative frequency (how often a word has been encountered throughout an individual's life) as well as the frequency trajectory (the fact that late acquired words almost by definition have higher frequencies in adults than in children, e.g., Bonin, Barry, Méot, \& Chalard, 2004; Cuetos \& Barbón, 2006; Ghyselinck, Lewis, \& Brysbaert, 2004; Juhasz, Yap, Raoul, \& Kaye, 2019; Pérez, 2007). These findings suggest that AoA might capture what frequency represents, but the reverse may not be necessarily true.

Our new measure of calculating PND (i.e., bPND) has theoretical implications for models of language production. Specifically, we argue that the existing measures of phonological neighborhoods do not capture all of the variation in the phonological network. Measures of neighborhood size may miss some phonological overlap with nonneighbors that share phonological features. While it is possible that PLD and PND measures are similar, this is less likely to be true of longer, more naturalistic samples of language, where a broader assessment of phonological networks may be warranted. However, it should be noted that bPND was more strongly correlated with neighborhood size measures (Phono_N and Phono_N_H) than with the phonological similarity measure (PLD), which suggests that near neighbors may constitute the largest influence on the effects we observed. This is consistent with previous research that has shown that minimal pairs (i.e., words with a phonological neighbor that differs by only one phonological feature such as voicing) influence articulatory patterns such as voice-onset times (VOTs; BaeseBerk \& Goldrick, 2009; Fricke, Baese-Berk, \& Goldrick, 2016; Nelson \& Wedel, 2017). Our results add to this literature by showing that the "nearness" effect of neighbors, and therefore their ultimate effect on a target's retrieval, is a function of the interaction between target and neighbor activation, rather than sheer distance.

\section{bPND $\times$ name agreement interaction}

In addition to interacting with frequency, bPND also interacted with name agreement. Although this effect was significant across all items, the facilitatory effect of bPND was larger for low-name agreement pictures. These results are consistent with broader research showing that PND affects lexical retrieval (Middleton \& Schwartz, 2010; Stemberger, 2004; Vitevitch, 2002a; Vitevitch \& Sommers, 2003). Specifically, our results show that when there are multiple different names for a certain picture, bPND facilitated retrieval, perhaps via interactive activation back to lexical nodes or increased lateral inhibition, which increases phonological accessibility and therefore the ultimate selection of the target word (e.g., Dell 1986, 1988). However, our results also show that the effect of bPND varied depending on the intensity of lexical competition showing a greater effect when competition is high. This finding is consistent with previous research showing that PND can affect higher-order language processes such as grammatical choices made during language production. For example, in one study, Hoover, Storkel, and Rice (2012) showed that the probability of using the incorrect infinite form of verbs (such as in "Abby walk to school yesterday") is lower for high- relative to low-PND verbs. The authors argued that this could be because high-PND verbs have richer lexical representations and such rich representations facilitate accessing pathways to higher-order processes such as finiteness marking (also see Hoover \& Storkel, 2013, for similar results). If PND can facilitate syntactic processing, it might also influence lexical selection when lexical competition is high (i.e., when name agreement is low). Importantly, in the case of highname agreement pictures where there is only one, or very few, suitable labels for a picture, the facilitatory effect of PND may be obviated, presumably because there is less or little lexical competition. Consistent with this hypothesis, variability in naming times was larger for low-name agreement items. Similar to the interaction between PND and AoA, our finding regarding the interaction between bPND and name agreement might also provide an explanation for the inconsistent PND effects reported by past studies. Specifically, given the varying effect of bPND across levels of name agreement, it could be the case that previous studies reporting inconsistent effects of PND on word production sampled high-name agreement pictures, potentially limiting the influence of PND.

Note that we did not observe a main effect of bPND, or any interactions between bPND and AoA or name agreement in the accuracy analysis (see Online Supplementary Material). One reason for this could be that accuracy reflects the end product of word retrieval whereas RTs reflect more of the processes involved in word retrieval (the more processes 
and/or the more complicated the processes involved in retrieving a target word, the longer the associated RT). Plus, the null effect of lexical variables such as PND for accuracy data is consistent with numerous previous studies (e.g., Sadat et al., 2014)

\section{Consistency with prior findings}

Our results were largely consistent with prior research. Specifically, we replicated the main effects of AoA (e.g., Carroll \& White, 1973; Gilhooly \& Gilhooly, 1979), frequency (Alario et al., 2004; Gordon \& Kurczek, 2014; Oldfield \& Wingfield, 1965; Sadat et al., 2014), name agreement (e.g., Middleton \& Schwartz, 2010; Stemberger, 2004; Vitevitch, 2002a; Vitevitch \& Sommers, 2003), and visual complexity (e.g., Attneave, 1957; Humphreys, Riddoch, \& Quinlan, 1988). However, we did not replicate previous findings showing a facilitatory effect of highly probable sound sequences on lexical processing (i.e., phonotactic probability, Edwards, Beckman, \& Munson, 2004; Vitevitch, Armbrüster, \& Chu, 2004). A number of differences in experimental design between the current and past studies could be driving this discrepancy. For example, Vitevitch et al. (2004) employed monosyllabic words with a CVC phonological format, and their procedure involved a familiarization phase in which participants practiced picture naming before the main experiment took place. Similarly, Vitevitch et al. (1997), Vitevitch and Luce (1998), and Edwards et al. (2004) employed nonwords rather than real words. Finally, and most importantly, past research has shown that the effect of phonotactic probability disappears when lexical variables such as neighborhood density are taken into consideration (e.g., Vitevitch \& Luce, 1998). Thus, while this discrepancy could have risen from a number of factors, the fact that we had numerous lexical variables including bPND in our statistical models makes the non-significant effect of phonotactic probability less surprising.

\section{Conclusion}

We observed that the effect of phonological neighborhood density on word production depends on the age at which a target word was acquired, suggesting that bPND's effect cannot be understood in isolation. Our results make important theoretical contributions to the current models of language production. Specifically, our results clearly demonstrate that the interaction between lexical and phonological representations (e.g., Dell, 1986, 1988) is heavily influenced by the relative activation levels of the target's phonological representations and its neighbors' phonological representations. In other words, the precise effect of the bilateral feedback between lexical and phonological representations depends on the relative activation of the target word's phonological features to that of its phonological neighbors. We also observed that the effect of a word's phonological neighborhood depends on name agreement such that it facilitates naming more when there is increased lexical competition (i.e., low name agreement). Overall, our results highlight how phonological characteristics of a neighborhood interact with lexical characteristics of the word (i.e., frequency) and selection demands of the environment (i.e., name agreement).

Acknowledgements This work was supported by a grant from the National Institutes of Health, National Institute on Aging, NIH R01 AG034138 (mtd). The content is solely the responsibility of the authors and does not necessarily represent the official views of the funding agencies. The information presented in this article has not previously been disseminated in other forms, including conference presentations. We thank the staff and scientists at the Center for Language Science, where the experiment was conducted, for their support.

Open Practices Statement The materials, data, and analysis scripts are available through the Open Science Framework (https://osf.io/pdgs4/ files/). None of the experiments were preregistered.

\section{References}

Alario, F. X., Ferrand, L., Laganaro, M., New, B., Frauenfelder, U. H., \& Segui, J. (2004). Predictors of picture naming speed. Behavior Research Methods, Instruments, \& Computers, 36(1), 140-155.

Attneave, F. (1957). Physical determinants of the judged complexity of shapes. Journal of Experimental Psychology, 53, 221-227.

Baese-Berk, M., \& Goldrick, M. (2009). Mechanisms of interaction in speech production. Language and Cognitive Processes, 24(4), 527554.

Barr, D. J., Levy, R., Scheepers, C., \& Tily, H. J. (2013). Random effects structure for confirmatory hypothesis testing: Keep it maximal. Journal of memory and language, 68(3), 255-278.

Barry, C., Morrison, C. M., \& Ellis, A.W. (1997).Naming the Snodgrass and Vanderwart pictures: Effects of age of acquisition, frequency, and name agreement. Quarterly Journal of Experimental Psychology, 50A, 560-585.

Bates, D., Maechler, M., \& Bolker, B., 2011. lme4: Linear mixed-effects models using S4 classes. R package version 0.999375-39. <http:// CRAN.R-project.org/package $=$ lme4>.

Baus, C., Costa, A., \& Carreiras, M. (2008). Neighbourhood density and frequency effects in speech production: A case for interactivity. Language and Cognitive Processes, 23(6), 866-888.

Belke, E., Brysbaert, M., Meyer, A. S., \& Ghyselinck, M. (2005). Age of acquisition effects in picture naming: Evidence for a lexicalsemantic competition hypothesis. Cognition, 96(2), B45-B54.

Berman, M. G., Hout, M. C., Kardan, O., Hunter, M. R., Yourganov, G., Henderson, J. M., ..., Jonides, J. (2014). The perception of naturalness correlates with low-level visual features of environmental scenes. PloS one, 9(12), e114572.

Best, W. M. (1995). A reverse length effect in dysphasic naming: When elephant is easier than ant. Cortex, 31, 637-652.

Bonin, P., Barry, C., Méot, A., \& Chalard, M. (2004). The influence of age of acquisition in word reading and other tasks: A never ending story? Journal of Memory and Language, 50, 456-476. 
Borowsky, R., \& Masson, M. E. (1999). Frequency effects and lexical access: On the interpretation of null pseudohomophone base-word frequency effects. Journal of Experimental Psychology: Human Perception and Performance, 25(1), 270.

Britt, A. E., Ferrara, C., \& Mirman, D. (2016). Distinct effects of lexical and semantic competition during picture naming in younger adults, older adults, and people with aphasia. Frontiers in psychology, 7, 813.

Brodeur, M. B., Dionne-Dostie, E., Montreuil, T., \& Lepage, M. (2010). The Bank of Standardized Stimuli (BOSS), a new set of 480 normative photos of objects to be used as visual stimuli in cognitive research. PloS one, 5(5), e10773.

Brodeur, M. B., Guerard, K., \& Bouras, M. (2014). Bank of Standardized Stimuli (BOSS) phase II: 930 new normative photos. PLoS One, 9(9), e106953.

Brown, G. D. A., \& Watson, F. L. (1987). First in, first out: Word learning age and spoken word frequency as predictors of word familiarity and word naming latency. Memory \& Cognition, 15, 208-216.

Carroll, J. B., \& White, M. N. (1973). Word frequency and age of acquisition as determiners of picture-naming latency. The Quarterly Journal of Experimental Psychology, 25(1), 85-95.

Chen, Q., \& Mirman, D. (2012). Competition and cooperation among similar representations: Toward a unified account of facilitative and inhibitory effects of lexical neighbors. Psychological review, 119(2), 417

R Core Team (2018). R: A Language and Environment for Statistical Computing. R Foundation for Statistical Computing, Vienna. https://www.R-project.org

Cuetos, F., \& Barbón, A. (2006). Word naming in Spanish. The European Journal of Cognitive Psychology, 18, 415-436.

Dell, G. S. (1986). A spreading-activation theory of retrieval in sentence production. Psychological Review, 93(3), 283-321.

Dell, G. S. (1988). The retrieval of phonological forms in production: Tests of predictions from a connectionist model. Journal of Memory and Language, 27(2), 124-142.

Dell, G. S., \& Gordon, J. K. (2003). Neighbors in the lexicon: Friends or foes. Phonetics and phonology in language comprehension and production: Differences and similarities, 6, 9-37.

Edwards, J., Beckman, M. E., \& Munson, B. (2004). The interaction between vocabulary size and phonotactic probability effects on children's production accuracy and fluency in nonword repetition. Journal of Speech, Language, and Hearing Research, 57, 421-436.

Ellis, A. W. \& Lambon Ralph, M.A. (2000). Age of acquisition effects in adult lexical processing reflect loss of plasticity in maturing systems: Insights from connectionist networks. Journal of Experimental Psychology: Learning, memory, and cognition, 26(5), 1103.

Forster, K. I. (1976). Accessing the mental lexicon. In R. J. Wales \& E. C. T. Walker (Eds.), New approaches to language mechanisms (pp. 257-287). Amsterdam: North-Holland.

Frank, A. F., Tanenhaus, M. K., Aslin, R. N., \& Salverda, A. P. (2007). Frequency, neighborhood density, and phonological similarity effects in picture naming: An artificial lexicon study. In Proceedings 29 annual conference on Cognitive Science Society, 995-1000.

Fricke, M., Baese-Berk, M. M., \& Goldrick, M. (2016). Dimensions of similarity in the mental lexicon. Language, Cognition and Neuroscience, 31(5), 639-645.

Ghyselinck, M., Lewis, M. B., \& Brysbaert, M. (2004). Age of acquisition and the cumulative-frequency hypothesis: A review of the literature and a new multi-task investigation. Acta Psychologica, 115, 43-67.

Gilhooly, K. J., \& Gilhooly, M. L. (1979). Age-of-acquisition effects in lexical and episodic memory tasks. Memory \& Cognition, 7(3), 214223.

Gilhooly, K. J., \& Watson, F. L. (1981). Word age-of-acquisition effects: A review. Current Psychological Reviews, 1(3), 269-286.
Goldinger, S. D., Luce, P. A., \& Pisoni, D. B. (1989). Priming lexical neighbors of spoken words: Effects of competition and inhibition. Journal of Memory and Language, 28(5), 501-518.

Goldrick, M., Folk, J. R., \& Rapp, B. (2010). Mrs. Malaprop's neighborhood: Using word errors to reveal neighborhood structure. Journal of Memory and Language, 62(2), 113-134.

Gordon, J. K. (2002). Phonological neighborhood effects in aphasic speech errors: Spontaneous and structured contexts. Brain and Language, 82(2), 113-145.

Gordon, J. K., \& Kurczek, J. C. (2014). The ageing neighbourhood: phonological density in naming. Language, Cognition and Neuroscience, 29(3), 326-344.

Harley, T. A., \& Bown, H. E. (1998). What causes a tip-of-the-tongue state? Evidence for lexical neighbourhood effects in speech production. British Journal of Psychology, 89(1), 151-174.

Hoover, J. R., \& Storkel, H. L. (2013). Grammatical treatment and specific language impairment: Neighbourhood density \& third person singular-s. Clinical linguistics \& phonetics, 27(9), 661-680.

Humphreys, G. W., Riddoch, M. J., \& Quinlan, P. T. (1988). Cascade processes in pictureidentification. Cognitive Neuropsychology, 5(1), $67-103$

Izura, C., Pérez, M. A., Agallou, E., Wright, V. C., Marín, J., StadthagenGonzález, H., \& Ellis, A. W. (2011). Age/order of acquisition effects and the cumulative learning of foreign words: A word training study. Journal of Memory and Language, 64(1), 32-58.

James, L. E., \& Burke, D. M. (2000). Phonological priming effects on word retrieval and tip-of-the-tongue experiences in young and older adults. Journal of Experimental Psychology: Learning, Memory, and Cognition, 26(6), 1378.

Jescheniak, J. D., \& Levelt, W. J. (1994). Word frequency effects in speech production: Retrieval of syntactic information and of phonological form. Journal of Experimental Psychology: Learning, Memory, and Cognition, 20(4), 824.

Juhasz, B. J., Yap, M. J., Raoul, A., \& Kaye, M. (2019). A further examination of word frequency and age-of-acquisition effects in English lexical decision task performance: The role of frequency trajectory. Journal of Experimental Psychology: Learning, Memory, and Cognition, 45(1), 82.

Kardan, O., Demiralp, E., Hout, M. C., Hunter, M. R., Karimi, H., Hanayik, T., ..., Berman, M. G. (2015). Is the preference of natural versus man-made scenes driven by bottom-up processing of the visual features of nature?. Frontiers in psychology, 6, 471 .

Lachman, R., Shaffer, J. P., \& Hennrikus, D. (1974). Language and cognition: Effects of stimulus codability, name-word frequency, and age of acquisition on lexical reaction time. Journal of Verbal Learning \& Verbal Behavior, 13, 613-625.

Lambon Ralph, M. A., \& Ehsan, S. (2006). Age of acquisition effects depend on the mapping between representations and the frequency of occurrence: Empirical and computational evidence. Visual Cognition, 13(7-8), 928-948.

Landauer, T. K., \& Streeter, L. A. (1973). Structural differences between common and rare words: Failure of equivalence assumptions for theories of word recognition. Journal of Verbal Learning and Verbal Behavior, 12(2), 119-131.

Luce, P. A., \& Pisoni, D. B. (1998). Recognizing spoken words: The neighborhood activation model. Ear \& Hearing, 19(1), 1-36.

Luce, P. A., Goldinger, S. D., Auer, E. T., \& Vitevitch, M. S. (2000). Phonetic priming, neighborhood activation, and PARSYN. Perception \& psychophysics, 62(3), 615-625.

Madan, C. R., Bayer, J., Gamer, M., Lonsdorf, T. B., \& Sommer, T. (2018). Visual complexity and affect: ratings reflect more than meets the eye. Frontiers in psychology, 8, 2368.

McClelland, J. L., \& Rumelhart, D. E. (1981). An interactive activation model of context effects in letter perception: Part 1. An account of basic findings. Psychological Review, 88, 375-407. 
Middleton, E. L., \& Schwartz, M. F. (2010). Density pervades: An analysis of phonological neighbourhood density effects in aphasic speakers with different types of naming impairment. Cognitive Neuropsychology, 27(5), 401-427.

Mirman, D., \& Magnuson, J. S. (2008). Attractor dynamics and semantic neighborhood density: Processing is slowed by near neighbors and speeded by distant neighbors. Journal of Experimental Psychology: Learning, Memory, and Cognition, 34(1), 65-79.

Mirman, D., Kittredge, A. K., \& Dell, G. S. (2010). Effects of near and distant phonological neighbors on picture naming. In Proceedings of the Annual Meeting of the Cognitive Science Society 32(32).

Moreno-Martinez, F. J., \& Montoro, P. R. (2012). An ecological alternative to Snodgrass \& Vanderwart: 360 high quality colour images with norms for seven psycholinguistic variables. PLoS ONE, 7(5), e37527.

Morrison, C. M., Ellis, A. W., \& Quinlan, P. T. (1992). Age of acquisition, not word frequency, affects object naming, not object recognition. Memory \& Cognition, 20(6), 705-714.

Morton, J. (1969). Interaction of information in word recognition. Psychological Review, 76, 165-178.

Munson, B., Swenson, C. L., \& Manthei, S. C. (2005). Lexical and phonological organization in children: Evidence from repetition tasks. Journal of Speech, Language and Hearing Research, 48(1), 108

Navarrete, E., Pastore, M., Valentini, R., \& Peressotti, F. (2015). First learned words are not forgotten: Age-of-acquisition effects in the tip-of-the-tongue experience. Memory \& cognition, 43(7), 10851103.

Nelson, N. R., \& Wedel, A. (2017). The phonetic specificity of competition: Contrastive hyperarticulation of voice onset time in conversational English. Journal of Phonetics, 64, 51-70.

Oldfield, R. C., \& Wingfield, A. (1965). Response latencies in naming objects. Quarterly Journal of Experimental Psychology, 17(4), 273281.

Paap, K. R., McDonald, J. E., Schvaneveldt, R. W., \& Noel, R. W. (1987). Frequency and pronounceability in visually presented naming and lexical decision tasks. In M. Coltheart (Ed.), Attention and performance XII: The psychology of reading (pp. 221-243). Hillsdale, NJ: Erlbaum.

Paivio, A., Clark, J. M., Digdon, N., \& Bons, T. (1989). Referential processing: Reciprocity and correlates of naming and imaging. Memory \& Cognition, 17, 163-174.

Pérez, M. Á. (2007). Age of acquisition persists as the main factor in picture naming when cumulative word frequency and frequency trajectory are controlled. The Quarterly Journal of Experimental Psychology, 60(1), 32-42.

Perret, C., Bonin, P., \& Laganaro, M. (2014). Exploring the multiple-level hypothesis of AoA effects in spoken and written object naming using a topographic ERP analysis. Brain and language, 135, 20-31.

Psychology Software Tools, Inc. [E-Prime 3.0]. (2016). Retrieved from https://www.pstnet.com.

Rosenholtz, R., Li, Y., \& Nakano, L. (2007). Measuring visual clutter. Journal of vision, 7(2), 17-17.

Rubenstein, H., Garfield, L., \& Millikan, J. A. (1970). Homographic entries in the internal lexicon. Journal of Verbal Learning and Verbal Behavior, 9, 487-492.
Sadat, J., Martin, C. D., Costa, A., \& Alario, F. X. (2014). Reconciling phonological neighborhood effects in speech production through single trial analysis. Cognitive psychology, 68, 33-58.

Snodgrass, J. G., \& Vanderwart, M. (1980). A standardized set of 260 pictures: norms for name agreement, image agreement, familiarity, and visual complexity. Journal of experimental psychology: Human learning and memory, 6(2), 174.

Snodgrass, J. G., \& Yuditsky, T. (1996). Naming times for the Snodgrass and Vanderwart pictures. Behavior Research Methods, Instruments, \& Computers, 28, 516-536.

Spivey, M. (2007). The continuity of mind. New York, NY, US: Oxford University Press.

Stemberger, J. P. (2004). Neighborhood effects on error rates in speech production. Brain and Language, 90, $413-422$.

Tabak, W., Schreuder, R., \& Baayen, R. H. (2010). Producing inflected verbs: A picture naming study. The Mental Lexicon, 5(1), 22-46.

Taler, V., Aaron, G. P., Steinmetz, L. G., \& Pisoni, D. B. (2010). Lexical neighborhood density effects on spoken word recognition and production in healthy aging. Journals of Gerontology. Series B, Psychological Sciences and Social Sciences, 65B(5), 551-560.

Tsai, P. T. (2018). Phonological neighborhood effect in spontaneous speech in adults who stutter. Journal of fluency disorders, 58, 86-93.

Vitevitch, M. S. (1997). The neighborhood characteristics of malapropisms. Language and Speech, 40, 211-228.

Vitevitch, M. S. (2002a). The influence of phonological similarity neighborhoods on speech production. Journal of Experimental Psychology. Learning, Memory, and Cognition, 28(4), 735-747.

Vitevitch, M. S. (2002b). Naturalistic and experimental analyses of word frequency and neighborhood density effects in slips of the ear. Language and speech, 45(4), 407-434.

Vitevitch, M. S., \& Luce, P. A. (1998). When words compete: Levels of processing in perception of spoken words. Psychological Science, 9(4), 325-329.

Vitevitch, M. S., \& Luce, P. A. (2016). Phonological neighborhood effects in spoken word perception and production. Annual Review of Linguistics, 2, 75-94.

Vitevitch, M. S., \& Sommers, M. S. (2003). The facilitative influence of phonological similarity and neighborhood frequency in speech production in younger and older adults. Memory \& cognition, 31(4), 491-504.

Vitevitch, M. S., \& Stamer, M. K. (2006). The curious case of competition in Spanish speech production. Language and Cognitive Processes, 21(6), 760-770.

Vitevitch, M. S., Luce, P. A., Charles-Luce, J., \& Kemmerer, D. (1997). Phonotactics and syllable stress: Implications for the processing of spoken nonsense words. Language and Speech, 40, 47-62.

Vitevitch, M. S., Armbrüster, J., \& Chu, S. (2004). Sublexical and lexical representations in speech production: Effects of phonotactic probability and onset density. Journal of Experimental Psychology: Learning, Memory, and Cognition, 30(2), 514.

Vitkovitch, M., \& Tyrrell, L. (1995). Sources of disagreement in object naming. Quarterly Journal of Experimental Psychology, 48A, 822848.

Publisher's note Springer Nature remains neutral with regard to jurisdictional claims in published maps and institutional affiliations. 